\title{
Cardiovascular Manifestations of Hyperthyroidism
}

\section{Rashmi Aggarwal ${ }^{1 *}$ and Pradeep Chugh ${ }^{2}$}

${ }^{1}$ Department of Thyroid and Endocrine Research, Institute of Nuclear Medicine and Allied Sciences-INMAS, Delhi, India

${ }^{2}$ Department of Cardiology, Institute of Nuclear Medicine and Allied Sciences-

INMAS, Delhi, India

*Corresponding Author: Rashmi Aggarwal, Department of Thyroid and Endocrine Research, Institute of Nuclear Medicine and Allied Sciences-INMAS, Delhi, India.
Received: July 20, 2021

Published: July 29, 2021

(C) All rights are reserved by Rashmi

Aggarwal and Pradeep Chugh.

\begin{abstract}
Hyperthyroidism is associated with increased morbidity and mortality from cardiovascular disease [1]. Thyroid hormones significantly affect the cardiovascular systems. Both Heart and blood vessels are affected through genomic and non-genomic mechanisms. Tri iodothyronine (T3) the active thyroid hormone directly initiates the transcription of specific and non-specific genes acting on THR in the nucleus [2].

Non genomic effects of T3 are exerted on the plasma membrane, mitochondria and sarcoplasmic reticulum of cardiac myocytes.

Hyperthyroidism causes a spectrum of hemodynamic changes in the cardiovascular system like increase in heart rate, cardiac output, stroke volume, myocardial contractility and Left Ventricular mass [3]. Atrial fibrillation is a common cardiac manifestation of hyperthyroidism. Even subclinical hyperthyroidism has been also associated with an increased risk of atrial fibrillation [4].

In patients with pre - existing heart disease, the hyperdynamic circulation accompanying hyperthyroidism will further impair cardiac function leading on to congestive cardiac failure, worsening angina, atrial fibrillation and thromboembolism [5].
\end{abstract}

Keywords: Hyperthyroidism; Heart Rate; Atrial Fibrillation

Haemodynamic changes accompanying hyperthyroidism

Hemodynamic effects of hyperthyroidism are due to direct effects of thyroid hormones on the heart and blood vessels. Hyperthyroidism causes an increase in resting heart rate, systolic blood pressure, blood volume, left ventricular mass, stroke volume, left ventricular ejection fraction and cardiac output [6]. Excessive amounts of circulating thyroid hormones lead on to increased myocardial oxygen demand [7]. Increased stoke volume and increased heart rate leads on to a massive increase in cardiac output $[7,8]$. The cardiac output may be increased to $250 \%$.

Apart from direct effects of thyroid hormones on cardiac musculature and functions, some important changes are seen in pe- ripheral circulation as well. Triiodothyronine (T3) causes relaxation of the vascular arterial smooth muscles cells leading on to vasodilatation and fall in systemic vascular resistance. Thyroid hormones also increase the generation of NO by the endothelial cells contributing to vasodilatation and further fall in systemic vascular resistance. In addition, excess thyroid hormone in hyperthyroid patient increases the metabolic rate which leads to increased oxygen demand of the peripheral vascular tissue. The fall in peripheral vascular resistance further increases the heart rate and also increases the blood flow to certain organs like skin, skeletal muscles and heart. Peripheral vasodilatation leads to fall in diastolic pressure and a consequent widening of pulse pressure. 
Cardiac manifestations in overt hyperthyroidism

Palpitation is the most important cardiac symptom and occurs in $85 \%$ of patients suffering from hyperthyroidism. Palpitations occur due to an increase in heart rate as well as an increase in myocardial contractility [9]. Dyspnoea on exertion and exercise intolerance occurs in $50 \%$ of patients. They occur due to inability of the patient to raise the cardiac output and also due to weakness of skeletal and respiratory muscles.

Cardiac auscultation reveals a first heart sound which is frequently accentuated and an accentuated pulmonic component of second heart sound. Systolic murmurs are frequently auscultated. These murmurs occur when increased amount of blood is rapidly expelled across the aortic valve. In addition, systolic murmurs indicating Tricuspid regurgitation or mitral regurgitation can also be noted. These systolic murmurs usually disappear once the euthyroid state is restored. In patients with Graves` disease there is an increased incidence of mitral valve prolapse [10].

\section{Rhythm disturbances in hyperthyroidism}

The most common rhythm abnormality in hyperthyroidism in sinus tachycardia. A resting heart rate of more than $90 / \mathrm{min}$ is seen in approximately $90 \%$ of patients suffering from hyperthyroidism [11].

Atrial fibrillation is the most common cardiac arrhythmia associated with hyperthyroidism. It occurs in $10-15 \%$ of patients with hyperthyroidism [12]. It is more common in males, and its incidence increases with increasing age [13]. Apart from atrial fibrillation, atrial flutter and paroxysmal supraventricular tachycardia can also occur in patients suffering from hyperthyroidism. Ventricular tachycardias rarely occur, but when they occur, they suggest the presence of an underlying heart disease which also needs to be investigated.

Atrial fibrillation is a major cause of morbidity and mortality in elderly patients with hyperthyroidism. It may lead on to heart failure and/or thromboembolic events especially in elderly patients and those suffering from other co morbid conditions. It is important to note here that even sub clinical hyperthyroidism can cause atrial fibrillation [14].

\section{Hypertension}

Hyperthyroidism is accompanied with systolic hypertension, more so in elderly population. Systolic hypertension is due to the inability of the vasculature to respond to the increased stroke vol- ume. Hyperthyroidism is associated with a marked reduction in the peripheral vascular resistance which leads on to a fall in diastolic blood pressure [15]. Restoration of euthyroid status in these toxic patients will lead to normalization of systolic hypertension.

Pulmonary hypertension and right ventricular dysfunction associated with hyperthyroidism

There have been scattered reports of thyrotoxicosis being associated with pulmonary hypertension, right ventricular failure and tricuspid regurgitation. These cardiovascular findings revert back to normal once the patient becomes euthyroid. The possible reasons for isolated right ventricular failure could be increased blood volume leading on to right ventricular overload, partly due to increased cardiac output and venous return [16]. The hyper dynamic circulation leads to increased pulmonary arterial pressure and right ventricular pressure [17]. As a result of which right ventricle becomes dilated and causes functional tricuspid regurgitation. Once tricuspid regurgitation occurs, the increased volume overload causes further dilatation of the tricuspid annulus. It is important for us to remember that when we come across a case of isolated and unexplained tricuspid regurgitation and right heart failure, hyperthyroidism should always be considered in the differential diagnosis.

\begin{tabular}{|c|c|}
\hline $\begin{array}{c}\text { Serial } \\
\text { No }\end{array}$ & $\begin{array}{c}\text { Haemodynamic changes in patients suffering from } \\
\text { hyperthyroidism }\end{array}$ \\
\hline 1. & $\begin{array}{c}\text { Increase in heart rate (due to increased symphathetic } \\
\text { tone) }\end{array}$ \\
\hline 2. & Increase in cardiac output \\
\hline 3. & $\begin{array}{c}\text { Increase in stroke volume (due to increase in preload } \\
\text { and reduction in afterload) }\end{array}$ \\
\hline 4. & Increase in myocardial contractility \\
\hline 5. & Peripheral vasodilation \\
\hline 6. & Decreased renal perfusion \\
\hline 7. & Reduction in peripheral vascular resistance \\
\hline
\end{tabular}

Table 1: Haemodynamic changes accompanying hyperthyroidism.

\section{Conclusion}

Hyperthyroidism is accompanied by profound alterations in the circulatory system. There is an increase in total blood volume, stroke volume along with a decrease in systemic vascular resistance and an increase in basal metabolic rate together resulting in a hyper dynamic circulation [18]. Untreated or severe hyperthyroidism may be associated with complications like congestive cardiac 
failure, atrial fibrillation or exacerbation of angina. Restoration of euthyroid state is of utmost importance in treating such patients. Sinus tachycardia and atrial fibrillation are the most frequent cardiovascular signs of thyrotoxicosis, and all elderly patients with atrial fibrillation should always be tested for thyroid function tests to rule out overt as well as subclinical hyperthyroidism.

\section{Bibliography}

1. Franklyn JA., et al. "Mortality after the treatment of hyperthyroidism after radioactive iodine". The New England Journal of Medicine 338 (1998): 712-718.

2. Dillman WH. "Biochemical basis of thyroid hormone action in the heart”. The American Journal of Medicine 88 (1990): 626630 .

3. Woeber KA. "Thyrotoxicosis and the heart". The New England Journal of Medicine 327 (1992): 94-98.

4. Marqusee E., et al. "Subclinical thyrotoxicosis". Endocrinology and Metabolism Clinics of North America 27 (1998): 37-49.

5. Ikram H. "The nature and prognosis of thyrotoxic heart disease". QJ Medicine 54 (1985): 19-28.

6. Klein I and Ojamaa K. "Thyroid hormones and the cardiovascular system". The New England Journal of Medicine 334 (2001): 501-509.

7. Fadel BM., et al. "Hyperthyroid heart disease". Clinical Cardiology 23 (2000): 402-408.

8. Polikar R., et al. "The thyroid and the heart". Circulation 87 (1993): 1435-1441.

9. Klein I and Ojamaa K. "Thyrotoxicosis and the heart". Endocrinology and Metabolism Clinics of North America 27 (1998): 51-62.

10. Brauman A., et al. "Mitral valve prolapse in hyperthyroidism of two different origins". British Heart Journal 53 (1985): 374377.

11. Panagoulis C., et al. "Hyperthyroidism and the heart". Hellenic Journal of Cardiology 49 (2008): 169-175.

12. Agner T., et al. "A re-evaluation of atrial fibrillation in thyrotoxicosis". Danish Medical Bulletin 31 (1948): 157-159.
13. Vardas PE and Mavrakis HE. "Atrial fibrillation: a symptom treated as a disease?". Hellenic Journal of Cardiology 47 (2006): 191-193.

14. Sawin CT., et al. "Low serum thyrotropin concentration as a risk factor for atrial fibrillation in older persons". The New England Journal of Medicine 331 (1994): 1249-1252.

15. Saito I and Saruta T. "Hypertension in thyroid disorders". Endocrinology and Metabolism Clinics of North America 23 (1994): 379-386.

16. Thumheer R., et al. "Hyperthyroidism and pulmonary hypertension". Journal of Internal Medicine 242 (1997): 185-188.

17. Xenopoulos NP., et al. "Severe right heart failure in a patient with Graves`disease”. Clinical Cardiology 19 (1996): 903-905.

18. Kenneth A and Woeber MD. "Thyrotoxicosis and the heart". The New England Journal of Medicine 327 (1992): 94-98.

\section{Volume 3 Issue 8 August 2021 (C) All rights are reserved by Rashmi Aggarwal and Pradeep Chugh.}

